\title{
Gerenciamento de enfermagem em serviço hospitalar de emergência: revisão integrativa da literatura
}

\section{Nursing management in hospital emergency service: integrative review of the literature}

\author{
Renata Cristina Antonelli ${ }^{1}$; José Aparecido Bellucci Junior ${ }^{2}$
}

\begin{abstract}
Resumo
Trata-se de uma revisão integrativa da literatura, que tem por objetivo analisar artigos científicos que versam a respeito da atuação do enfermeiro no gerenciamento em serviço hospitalar de emergência. As bases de dados utilizadas foram Literatura Latino-Americana e do Caribe em Ciências da Saúde (LILACS), Medical Literature Analysis and Retrieval System Online (MEDLINE), Integrated Building Environmental Communications System (IBECS), Scientific Electronic Library Online (SCIELO) e Base de dados de enfermagem (BDENF). Foram selecionados 10 artigos para realização da pesquisa, os quais foram classificados em duas categorias temáticas, a saber: "analisando o trabalho do enfermeiro no serviço hospitalar de emergência" e "organizando o trabalho do enfermeiro no serviço hospitalar de emergência". A primeira categoria abordou as funções do enfermeiro e a satisfação desses profissionais, e a segunda, os problemas identificados no serviço hospitalar de emergência e as estratégias existentes para organizar o serviço, respectivamente. Conclui-se que o tema desenvolvido mostrou como se apresenta o trabalho do enfermeiro como gestor desse serviço, além de diferentes estratégias existentes capazes de atenuar ou eliminar os problemas identificados nas unidades de urgência e emergência de serviços hospitalares.
\end{abstract}

Palavras-chave: Serviço hospitalar de emergência. Enfermagem em emergência. Gestor de saúde.

\begin{abstract}
This is an integrative literature review, which aims to analyze scientific articles that discuss the work of nurses in the management of hospital emergency services. The databases used were Latin American and Caribbean Health Science Literature (LILACS), Medical Literature Analysis and Retrieval System Online (MEDLINE), Integrated Building Environmental Communications System (IBECS), Scientific Electronic Library Online (SciELO) and Nursing Database (BDENF). To conduct the survey, 10 articles were selected and classified into two themes, namely: "analyzing the work of the nurse in hospital emergency services" and "organizing the nurse work in hospital emergency services". The first category discussed the functions of the nurse and the satisfaction of these professionals, and the second, the problems identified in the hospital emergency service and strategies to organize the service, respectively. The theme developed showed how the work of the nurse presents as manager of that service, in addition to various strategies to alleviate or eliminate the identified problems in the urgency and emergency units of the hospital services.
\end{abstract}

Key words: Emergency hospital service. Emergency nursing. Health manager.

\footnotetext{
${ }^{1}$ Especialista em Urgência e Emergência pela Faculdade de Ciências Médicas da Santa Casa de São Paulo; Enfermeira em Unidade de Terapia Intensiva do Hospital Santa Marcelina. E-mail: re-antonelli@hotmail.com

${ }^{2}$ Doutorando em Enfermagem pela Universidade Estadual de Maringá. Docente na Universidade Estadual do Norte do Paraná. E-mail: bellucci@uenp.edu.br
} 


\section{Introdução}

$\mathrm{Na}$ atualidade, frente à importância que o enfermeiro tem ocupado no gerenciamento da equipe de enfermagem nas instituições de saúde, há de se destacar que o desenvolvimento de conhecimentos, habilidades e atitudes são exigências à atuação desse profissional na promoção da saúde (LOURENÇÃO; BENITO, 2010) e gestão dos serviços.

Em 1860, Florence Nightingale já destacava a importância do enfermeiro no gerenciamento das ações de enfermagem ao categorizar os profissionais em Nurses e Lady-Nurses, diferenciando os deveres. As Nurses eram as responsáveis pela execução de tarefas manuais e as Lady-Nurses pelo ensino e supervisão do trabalho (SOUZA et al., 2006).

No contexto mundial, mais específicamente nos Estados Unidos, foi criado um molde para o Gerenciamento de Enfermagem (GE), em que abordam as seguintes aptidões: poder pessoal; efetividade interpessoal; gestão de recursos humanos; gestão financeira; cuidados com a equipe, com o paciente e consigo mesmo; e pensamento sistematizado (NURSING LEADERSHIP INSTITUTE, 2003).

No Brasil, a atuação do enfermeiro é regulamentada pela Lei $\mathrm{n}^{0} 7.498$, de 25 de junho de 1986 que no Art. $11^{\circ}$ cita as ações privativas do enfermeiro gestor: a direção e chefia dos órgãos de enfermagem nas instituições públicas ou privadas; a organização das atividades técnicas e auxiliares; e o planejamento, coordenação, execução e avaliação dos serviços prestados pela enfermagem, entre outros (JORGE et al., 2007).

Apesar de as especificações da lei supra citada serem privativas do enfermeiro, é possível destacar que nos serviços hospitalares a prática gerencial carece de organização, planejamento, conhecimentos e competência para coordenar, o que pode dificultar o desempenho mais efetivo de tais profissionais como agentes responsáveis pelo gerenciamento e desenvolvimento da sua equipe e da assistência ao cliente (SANTOS, 2010).

Ao pensar em qualidade no atendimento hospitalar, os Serviços Hospitalares de Emergência (SHE) se despontam como um dos setores mais críticos quanto ao GE por ocorrer nesse serviço a imprevisibilidade de ocorrências e ritmos altamente acelerados para $o$ atendimento. Acresça-se a esses fatores, a superlotação e a falta de recursos os quais interferem negativamente no planejamento e na padronização dos procedimentos prestados pela equipe de saúde (SANTOS, 2010).

Os SHE são unidades complexas no que diz respeito ao ambiente, ao usuário e aos profissionais que oferecem o serviço. Dessa forma, a equipe de enfermagem que trabalha nesse setor deve dispor de agilidade, habilidade, destreza e capacidade de raciocinar de maneira consciente e segura quanto ao paciente atendido, sem que o cuidado deixe de ser a ligação entre o profissional e o usuário.

Buscando identificar quais são as ações de gerenciamento em SHE desenvolvidas por enfermeiros, motivou-se a realizar esse estudo, que tem como pergunta norteadora: como se apresenta a fundamentação científica acerca da atuação do enfermeiro no gerenciamento em SHE?

Para responder a questão de pesquisa, esse estudo teve por objetivo analisar artigos científicos que versam a respeito da atuação do enfermeiro no gerenciamento em SHE.

Justifica-se a realização dessa pesquisa pelo fato de que a discussão sobre gerenciamento de enfermagem em SHE produz conhecimentos que poderão subsidiar a tomada de decisão dos enfermeiros à melhoria da qualidade dos processos assistenciais e gerenciais desses serviços. 


\section{Método}

Trata-se de uma revisão integrativa da literatura, a qual se caracteriza por um estudo de abordagem mais extensa relativo às revisões bibliográficas, em que se admite a inclusão de pesquisas experimentais e não-experimentais para o entendimento pleno do tema analisado (SOUZA; SILVA; CARVALHO, 2010). Em outras palavras, é a análise dos achados já existentes na literatura sobre um determinado assunto, em que é possível reunir e sintetizar de forma sistemática o conteúdo estudado (MENDES; SILVEIRA; GALVÃO, 2008).

Para a realização da revisão integrativa da literatura foram utilizadas seis fases: $1^{\text {a }}$ Fase - elaboração da pergunta norteadora; $2^{\mathrm{a}}$ Fase busca ou amostragem na literatura; $3^{\mathrm{a}}$ Fase - coleta de dados; 4 ${ }^{\mathrm{a}}$ Fase - análise crítica dos estudos incluídos; $5^{\mathrm{a}}$ Fase - discussão dos resultados; e $6^{\text {a }}$ Fase - apresentação da revisão integrativa (SOUZA; SILVA; CARVALHO, 2010).

A pergunta norteadora do estudo foi: Como se apresenta a fundamentação científica acerca da atuação do enfermeiro no gerenciamento de serviço hospitalar de emergência?

A busca na literatura foi realizada nas bases de dados LILACS, MEDLINE, IBECS, SCIELO e BDENF. O descritor utilizado para análise dos estudos contidos nas bases foi Emergency Nursing, disponível nos Descritores em Ciências da Saúde (DeCS).

Para a inclusão das publicações foram seguidos alguns critérios: textos completos, nos idiomas português, inglês ou espanhol, publicados entre os anos de 2006 e 2010, que tiveram resultados conclusivos sobre a atuação do enfermeiro na gestão de serviços hospitalares de emergência.

O título e o resumo das publicações foram analisados e aquelas que possuíam considerações importantes a respeito da atuação do enfermeiro no Serviço Hospitalar de Emergência foram selecionadas para que o artigo completo fosse lido na etapa seguinte.

Para coleta de dados foi aplicado um instrumento validado por Ursi (2005) aos artigos selecionados para pesquisa. Os itens observados foram: identificação; instituição sede do estudo; tipo de publicação; características metodológicas do estudo, dentre elas, tipo de publicação, objetivo ou questão de investigação, amostra, tratamento dos dados, intervenções realizadas, resultados, análise, implicações e nível de evidência; e avaliação do rigor metodológico (SOUZA; SILVA; CARVALHO, 2010).

\section{Resultados}

A quantidade de textos completos encontrados por meio do descritor Emergency Nursing nos bancos de dados utilizados foram de 949, que se apresentavam na seguinte disposição: LILACS (147), MEDLINE (497), IBECS (2), SCIELO (101), BDENF (202).

Ao considerar apenas os estudos publicados entre os anos de 2006 e 2010, 388 textos foram eliminados. Após a análise de títulos e resumos dos 561 estudos restantes, 465 não ofereciam dados conclusivos sobre o tema estudado ou não seguiam os critérios propostos; 81 estavam repetidos na literatura e, finalmente, 15 resumos foram aceitos para que seus artigos completos fossem lidos posteriormente.

A leitura dos artigos aceitos foi feita de forma exaustiva, o que resultou na recusa de mais cinco artigos, pois não apresentavam conclusões relacionadas ao assunto abordado.

Seguindo para a $3^{\text {a }}$ fase realizou a coleta de dados de cada um dos 10 artigos selecionados para a pesquisa.

No Quadro 1 estão especificados o título do artigo, o título do periódico, os autores e o ano de publicação de cada pesquisa. Os artigos foram 
nomeados de $\mathrm{A} 1$ à $\mathrm{A} 10$, em ordem de coleta e ano de publicação.

Quadro 1 - Identificação dos artigos selecionados que versam a respeito da atuação do enfermeiro no gerenciamento de serviço hospitalar de emergência. Bandeirantes, 2011

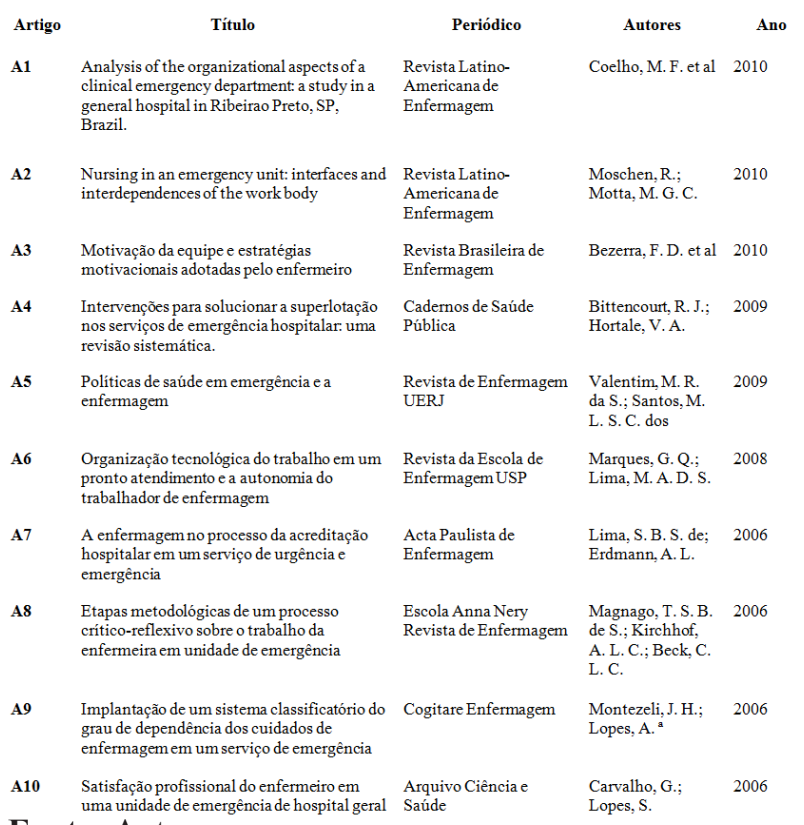

Fonte: Autores.

As distribuições dos artigos selecionados quanto ao ano de publicação foram: três artigos no ano de 2010, dois em 2009, um em 2008 e quatro em 2006, portanto, no ano de 2007 não houve publicação relevante sobre o tema estudado nas bases de dados analisadas.

Todas as publicações selecionadas são nacionais, sendo duas no idioma inglês e as demais em português.

$\mathrm{Na} 4^{\mathrm{a}}$ fase foi realizada a análise criteriosa dos dez artigos estudados, emanaram-se duas categorias temáticas: analisando o trabalho do enfermeiro no SHE e organizando o trabalho do enfermeiro no SHE.

$\mathrm{Na} 5^{\mathrm{a}}$ fase e na $6^{\mathrm{a}}$ fase foi possível destacar as discussões estabelecidas a partir da análise dos artigos selecionados para a pesquisa e apresentar os conceitos obtidos por meio da revisão integrativa da literatura.

Três artigos se enquadraram na categoria "analisando o trabalho do enfermeiro no SHE", pois destacam artigos que abordam as funções do enfermeiro e/ou a satisfação dos profissionais enfermeiros.

No Quadro 2 são apresentados o tipo de estudo, o objetivo, o resultado e a conclusão de cada artigo dessa categoria.

Quadro 2 - Caracterização dos estudos contidos na categoria temática "Analisando o Trabalho do Enfermeiro no SHE”. Bandeirantes, 2011

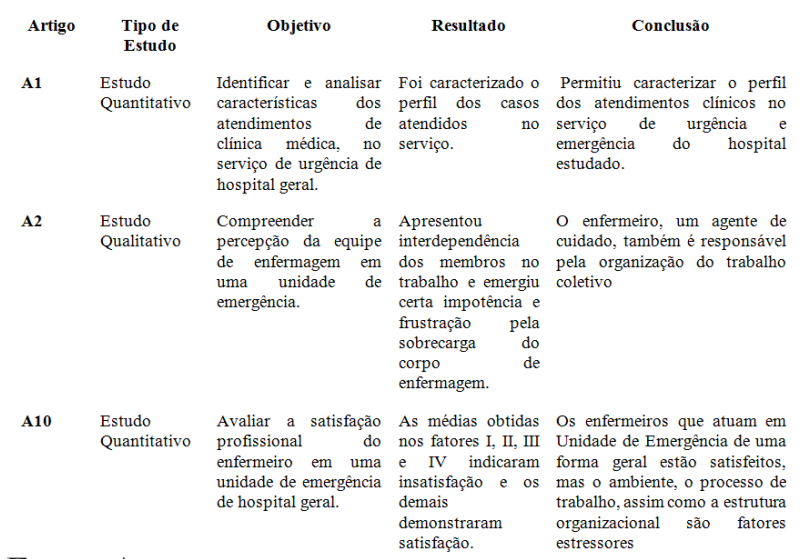

Fonte: Autores.

\section{Discussão}

Entre as funções que o enfermeiro desempenha no SHE destacam: o ato de cuidar, prever e prover recursos materiais e humanos, coordenar, articular e controlar as atividades da enfermagem, resolver problemas gerais e fazer a supervisão e o gerenciamento do serviço, entre outros [A1 (COELHO et al., 2010)].

Quanto ao gerenciamento do serviço, uma tese de doutorado apresentada em São Paulo, refere que o planejamento e a tomada de decisão, como funções atribuidas ao enfermeiro, auxiliam no desenvolvimento da gestão do serviço. Além disso, acrescenta que quanto às funções administrativas do enfermeiro, cita a liderança, a comunicação, a 
supervisão, a avaliação e a delegação (PERES, 2006).

Ainda com relação às ações realizadas pelo enfermeiro, o estudo A1 (COELHO et al., 2010) refere que, as atividades que são atribuídas a esse profissional no SHE requerem conhecimentos científicos, tecnológicos e de gestão específicos das unidades de urgência e emergência.

O estudo A1 (COELHO et al., 2010) refere que o trabalho do enfermeiro depende de suportes que podem auxiliar no planejamento do serviço oferecido ao usuário e sugere que a análise da demanda de atendimentos realizados em um hospital é um importante recurso de apoio ao serviço e aos profissionais envolvidos, pois pressupõe a quantidade de atendimentos que serão necessários nos diferentes dias da semana, nas horas do dia, entre outros, a fim de auxiliar na organização e na gestão dos atendimentos realizados. Além disso, dá subsídio aos enfermeiros para que eles possam oferecer um SHE compatível às necessidades dos usuários.

Com relação à satisfação dos profissionais da saúde, para que haja um bom relacionamento dos membros da equipe de trabalho, o estudo A2 (MOSCHEN; MOTTA, 2010) refere que a reflexão sobre a ação do grupo na assistência de enfermagem pode favorecer em um agradável ambiente de trabalho, pois a satisfação desse trabalhador, identificada nesses momentos, influencia a interação dele com os outros profissionais e seu desempenho.
Quanto à avaliação de algumas vertentes da satisfação, o estudo A10 (CARVALHO; LOPES, 2006) identificou, a saber: a satisfação geral, o pouco desgaste físico e psicológico e a localização da empresa. Ao passo que, as dimensões que indicaram insatisfação dos enfermeiros foram: a não relevância dada ao status da função, os escassos benefícios compensadores, o não reconhecimento e a falta de desenvolvimento pessoal.

Apesar da evolução existente quanto à satisfação dos enfermeiros demonstrada por meio do estudo A10 (CARVALHO; LOPES, 2006), ainda existem alguns pontos que causam insatisfação nesses profissionais e para que o status da profissão, os benefícios compensadores, o reconhecimento e o desenvolvimento pessoal passem a ser satisfatórios para os enfermeiros. Segundo o mesmo estudo, é necessário a mudança no processo de formação desses profissionais destacando o cuidado como objeto de estudo, além do reconhecimento desse profissional pelas demais categorias profissionais e pela comunidade.

Os outros sete artigos foram direcionados a categoria temática "organizando o trabalho do enfermeiro no SHE", pois destacam artigos que versam a respeito dos problemas identificados no SHE e/ou das estratégias existentes para organizar o processo de trabalho dos enfermeiros.

No Quadro 3 também são apresentados de forma sucinta o tipo de estudo, o objetivo, o resultado e a conclusão de cada artigo dessa categoria. 
Quadro 3 - Caracterização dos estudos contidos na categoria temática "Organizando o Trabalho do Enfermeiro no SHE”. Bandeirantes, 2011.

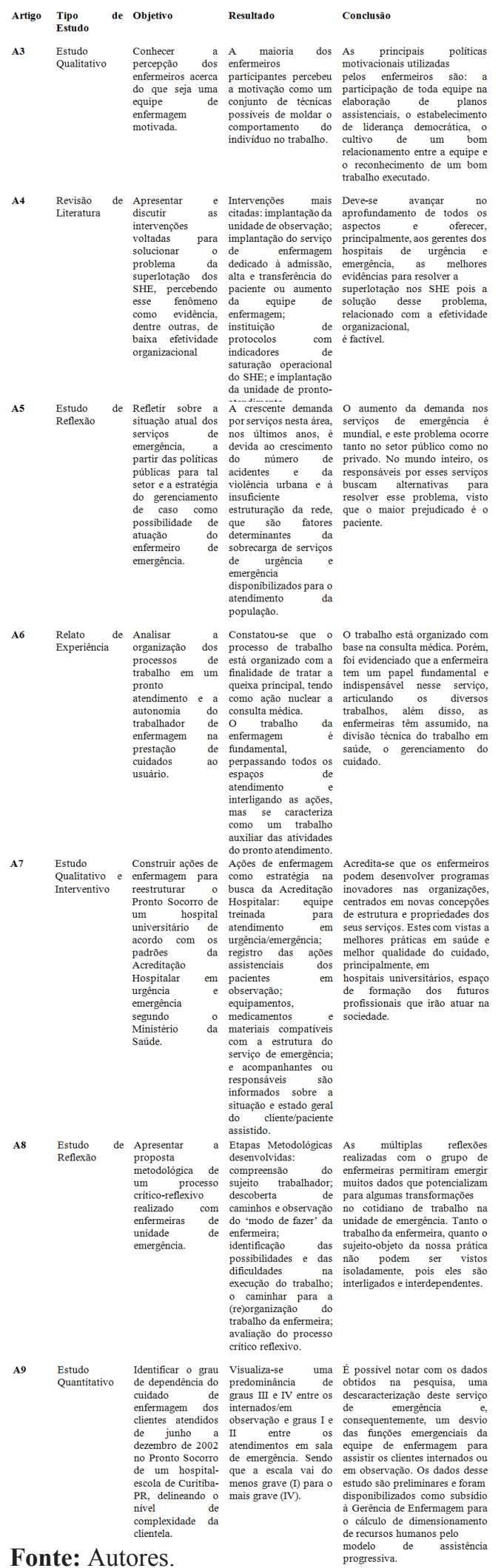

Primeiramente, antes de citar as intervenções necessárias para resolver ou atenuar os problemas no SHE, é possível identificar os problemas encontrados no serviço. Segundo o estudo A5 (VALENTIM; SANTOS, 2009), a incapacidade dos serviços básicos e secundários de saúde em realizar os primeiros atendimentos ao usuário e o aumento da demanda nos últimos anos, levam a sobrecarga dos serviços terciários, ou seja, os serviços de urgência e emergência e, tal sobrecarga, faz com que os pacientes com emergências reais esperem por horas para serem atendidos.

A literatura menciona que outra causa da sobrecarga dos SHE é o fato dos usuários utilizarem esse serviço como uma alternativa de fácil acesso e rápida resolução, isto é, as consultas, os medicamentos, os procedimentos, os exames laboratoriais e as internações acontecem em menor período de tempo, o que atrai a clientela e a faz por esse serviço sem que o atendimento tenha tal necessidade (MARQUES; LIMA, 2007).

Um problema identificado quanto a organização do processo de trabalho realizado pelo enfermeiro, segundo o estudo A6 (MARQUES; LIMA, 2008), é que verificou-se que o trabalho está direcionado no tratamento da queixa principal e, por vezes, tem como ação central o atendimento médico. Além disso, a pesquisa cita que a preocupação com o cumprimento das tarefas e a realização dos procedimentos torna o trabalho muito manual e repetitivo, o que aliena o trabalhador e o afasta da atuação fundamentada e científica.

Estudo realizado com enfermeiros da unidade de internação de um hospital privado defende que existem enfermeiros que têm um bom desempenho assistencial, mas não desempenham suas funções administrativas de forma adequada e vice-versa. Quem desenvolve bem suas funções administrativas, tem dificuldade em desenvolver ações assistências, o que prova que existe dificuldade em dimensionar simultaneamente ambas as partes, gerencial e assistencial. Dessa forma, o enfermeiro que realiza a 
dimensão administrativa valoriza sua atuação como função essencial para o cuidado e quem realiza o cuidado diminui as ações gerenciais (HAUSMANN; PEDUZZI, 2009).

O estudo A9 (MONTEZELI; LOPES, 2006) aponta que a maioria dos atendimentos de alta complexidade ocorre em salas de observação, enquanto os atendimentos realizados na sala de emergência são de menor complexidade. A situação apresentada ocorre, provavelmente, pela falta de leitos para internação, o que faz com que pacientes que necessitam de cuidados intensivos permaneçam internados em salas de observação e, por conseguinte, seu estado geral seja agravado por conta da espera e do serviço não destinado aos cuidados de internação.

O estudo A4 (BITTENCOURT; HORTALE, 2009) apresenta intervenções que envolvem os trabalhadores de enfermagem e que podem minimizar a superlotação do serviço, dentre elas: a implantação do serviço de enfermagem dedicado à admissão, alta e transferência do paciente; identificação dos pacientes que procuram o SHE com mais frequência e encaminhá-los à rede básica; capacitação das equipes da unidade de prontoatendimento do SHE; envolvimento dos gerentes hospitalares na identificação dos problemas e soluções para a superlotação no SHE; entre outras. Para solucionar a superlotação é necessária a organização efetiva do sistema, prova de que a participação dos gestores na resolutividade desse problema é indispensável.

O estudo A5 (VALENTIM; SANTOS, 2009) acrescenta que o problema da superlotação é mundial e que os responsáveis pelos serviços buscam, constantemente, alternativas para mudar essa situação, pois a espera agrava ainda mais o quadro clínico do usuário e, assim, aumentam as sequelas provenientes da patologia.

Para que encaminhamentos desnecessários não ocorram no SHE, e que seja atenuado o problema de superlotação, a Política Nacional de Atenção às
Urgências preconiza que as unidades de atenção básica, durante seu horário de funcionamento, devem estar disponíveis para resolver pequenas urgências da população de sua abrangência (BRASIL, 2006). Estes profissionais devem estar dispostos a assumir a co-responsabilidade da condição crônica, de forma que seja possível a prevenção da agudização dos casos atendidos que são eventos agudos das doenças crônicas, diferentemente das condições agudas, que são como um evento-sentinela que sinaliza falha na assistência prestada durante a condição crônica da patologia (MENDES, 2011).

Quando a condição da patologia for aguda, a atenção é prestada no pronto atendimento, seu início é rápido, a duração é curta, os profissionais devem selecionar e prescrever o tratamento e a resposta deve ser reativa e episódica, ao passo que, quando a condição for crônica, seu início é gradual, a duração é indefinida, a resposta é proativa e contínua e os profissionais devem educar e fazer parceria com o usuário, desenvolvendo plano de cuidados (MENDES, 2011).

Versando, ainda, sobre estratégias para modificar a situação atual dos SHE, alguns autores referem que os enfermeiros e médicos consideram que o número de profissionais deveria ser proporcional a quantidade de pacientes atendidos, pois mesmo com ritmo intenso, algumas atividades não são realizadas pela incapacidade de atender a demanda (GARLET et al., 2009).

Quando se trata de estratégias voltadas para a equipe de trabalho, o estudo A7 (LIMA; ERDMANN, 2006), cita alguns instrumentos que podem ser utilizados pelo serviço de enfermagem para manter a organização do setor: regimento interno, organograma, sistemas de comunicação, técnicas, rotinas e sistemas de controle. Ainda, o mesmo estudo, sugere algumas atividades que podem ser desenvolvidas pelo enfermeiro, em busca da acreditação hospitalar para que ocorra a atualização e a educação dos profissionais, dentre elas: treinamento da equipe para atender 
casos de urgência/emergência, registro das ações assistenciais dos pacientes em observação; utilização de equipamentos, medicamentos e materiais compatíveis com a estrutura do serviço; e proporcionar informações sobre a situação e o estado geral do cliente/paciente assistido aos acompanhantes ou responsáveis.

A reflexão realizada por enfermeiras no estudo A8 (MAGNAGO; KIRCHHOF; BECK, 2006) pôde dar suporte a tais profissionais para que elas realizem algumas transformações no processo de trabalho e nos seus elementos, ou seja, o objeto, a finalidade, os instrumentos e a força de trabalho. Em suma, quando ocorre uma mudança na conduta dos profissionais gestores da equipe, possibilita que os demais membros da equipe tenham mais facilidade de aderir à modificação e tornar a assistência mais qualificada.

Vale ressaltar que a motivação, analisada no estudo A3 (BEZERRA et al., 2010), também é parte integrante na organização do trabalho do enfermeiro no SHE, pois, por meio dela, é possível moldar as atitudes dos profissionais em serviço. Ademais, o mesmo estudo destaca que a motivação é influenciada pelo ambiente de trabalho organizado, bons salários, carga horária de trabalho reduzida e cursos de capacitação, portanto, é importante que as instituições e os gestores, junto à equipe de trabalho, pensem em alternativas que possam minimizar a desmotivação encontrada nesses profissionais, afinal, esse sentimento influi na eficiência dos serviços prestados e na qualidade de vida dos trabalhadores.

\section{Conclusão}

É possível afirmar que a realização desse trabalho permitiu obter viso Panorâmico sobre as ações de gerenciamento em SHE. Os artigos pesquisados responderam, positivamente, a pergunta norteadora do estudo, pois, através deles, verificou-se como se apresenta a gestão do SHE realizada pelo enfermeiro.
É necessário que os enfermeiros tenham preparo acadêmico e profissional para exercer as atividades atribuídas a esse profissional de forma correta e eficaz, a fim de que as ações sejam fundamentadas na ciência e não aconteçam de forma improvisada.

Conclui-se que as intervenções identificadas mais eficazes para que seja possível a melhoria do SHE e que envolvem os profissionais gestores do serviço, são: o encaminhamento de pacientes que necessitam de atenção primária à Rede Básica de Saúde, a fim de que possa atenuar o grande problema das superlotações nos pronto-socorros; a qualificação e o treinamento dos profissionais atuantes nas unidades de urgência e emergência, para que eles estejam preparados para receber pacientes com risco de morte e para que erros tornem-se nulos; a contratação de mais profissionais, para que a quantidade de trabalhadores seja compatível com a demanda; assim como, a reflexão sobre o objeto, a finalidade, os instrumentos e a força de trabalho e a motivação dos profissionais da enfermagem, para que eles possam trabalhar com mais prazer, satisfação e amor ao próximo.

É importante citar, também, como forma de estratégia para melhoria do SHE, a implantação do Acolhimento e classificação de risco nos serviços de emergência.

Sugere-se que pesquisas que avaliem a implantação das estratégias propostas por enfermeiros gestores possam colaborar para a valorização de tal profissional e possam revelar uma forma de reestruturação dos modelos de gestão no SHE.

\section{Referências}

BEZERRA, F. D.; ANDRADE, M. F. C.; ANDRADE, J. S.; VIEIRA, M. J.; PIMENTEL, D. Motivação da equipe e estratégias motivacionais adotadas pelo enfermeiro. Revista Brasileira de Enfermagem, Brasília, v. 63, n. 1, p. 33-37, jan./fev. 2010. 
BITTENCOURT, R. J.; HORTALE, V. A. Intervenções para solucionar a superlotação nos serviços de emergência hospitalar: uma revisão sistemática. Caderno de Saúde Pública, Rio de Janeiro, v. 25, n. 7, p. 1439-1454, jul. 2009.

BRASIL. Ministério da Saúde. Política Nacional de Atenção às Urgências. Brasília, 2006.

CARVALHO, G.; LOPES, S. Satisfação profissional do enfermeiro em uma unidade de emergência de hospital geral. Arquivo Ciência e Saúde, São José do Rio Preto, v. 13, n. 4, p. 215 219, out./dez. 2006.

COELHO, M. F.; CHAVES, L. D. P.; ANSELMI, M. L.; HAYASHIDA, M.; SANTOS, C. B. Analysis of the organizational aspects of a clinical emergency department: a study in a general hospital in Ribeirão Preto, SP, Brazil. Revista Latino-Americana de Enfermagem, Ribeirão Preto, v. 18, n. 4, p. 770-777, jul./ago. 2010.

GARLET, E. R.; LIMA, M. A. D. S.; SANTOS, J. L. G.; MARQUES, G. Q. Organização do trabalho de uma equipe de saúde no atendimento ao usuário em situações de urgência e emergência. Revista Texto \& Contexto-Enfermagem, Florianópolis, v. 18, n. 4, p. 266-272, abr./jun. 2009.

HAUSMANN, M.; PEDUZZI, M. Articulação entre as dimensões gerencial e assistencial do processo de trabalho do enfermeiro. Revista Texto \& Contexto-Enfermagem, Florianópolis, v. 18 , n. 2, p. 258-265, abr./jun. 2009.

JORGE, M. S. B.; FREITAS, C. H. A.; NÓBREGA, M. F. B.; QUEIROZ, M. V. Gerenciamento em enfermagem: um olhar crítico sobre o conhecimento produzido em periódicos brasileiros (2000-2004). Revista Brasileira de Enfermagem, Brasília, v. 60, n. 1, p. 81-86, jan./ fev. 2007.

LIMA, S. B. S. de; ERDMANN, A. L. A enfermagem no processo da acreditação hospitalar em um serviço de urgência e emergência. Acta Paulista de Enfermagem, São Paulo, v. 19, n. 3, p. 271-278, 2006.
LOURENCAO, D. C. de A.; BENITO, G. A. V. Competências gerenciais na formação do enfermeiro. Revista Brasileira de Enfermagem, Brasília, v. 63, $\mathrm{n}$. 1, p. 91-97, 2010.

MAGNAGO, T. S. B. S.; KIRCHHOF, A. L. C.; BECK, C. L. C. Etapas metodológicas de um processo crítico-reflexivo sobre o trabalho da enfermeira em unidade de emergência. Escola Anna Nery Revista de Enfermagem, Rio de Janeiro, v. 10, n. 2, p. 286-296, ago. 2006.

MARQUES, G. Q.; LIMA M. A. D. S. Demandas de usuários a um serviço de pronto atendimento e seu acolhimento ao sistema de saúde. Revista LatinoAmericana de Enfermagem, Ribeirão Preto, v. 15, n. 1, p. 13-19, jan./fev. 2007.

MARQUES, G. Q.; LIMA, M. A. D. S. Organização tecnológica do trabalho em um pronto atendimento e a autonomia do trabalhador de enfermagem. Revista da Escola de Enfermagem-USP, São Paulo, v. 42, n. 1, p. 41-47, mar. 2008.

MENDES, K. D. S.; SILVEIRA, R. C. C. P.; GALVÃO, C. M. Revisão integrativa: método de pesquisa para a incorporação de evidências na saúde e na enfermagem. Revista Texto \& ContextoEnfermagem, Florianópolis, v. 17, n. 4, p. 758-764, out./dez. 2008.

MENDES, E. V. As redes de atenção à saúde. Brasília: Organização Pan-Americana da Saúde, 2011.

MONTEZELI, J. H.; LOPES, A. A. Implantação de um sistema classificatório do grau de dependência dos cuidados de enfermagem em um serviço de emergência. Cogitare Enfermagem, Curitiba, v. 11, n. 3, p. 239-244, set./dez. 2006.

MOSCHEN, R.; MOTTA, M. G. C. Nursing in an emergency unit: interfaces and interdependences of the work body. Revista Latino-Americana de Enfermagem, Ribeirão Preto, v. 18, n. 5, p. 960-967, set./out. 2010.

NURSING LEADERSHIP INSTITUTE. The nursing leadership institute competency model. 2003. Disponível em: $<$ http://nursing.fau.edu/uploads/ docs/358/nursing_leadership_model2.pdf $>$. Acesso em: 12 nov. 2011. 
PERES, A. M. Competências gerenciais do enfermeiro: relação entre as expectativas da instituição formadora e do mercado de trabalho. 2006. 250fls. Tese (Doutorado em Enfermagem) Escola de Enfermagem, Universidade de São Paulo, São Paulo, 2006.

SANTOS, J. L. A dimensão gerencial do trabalho do enfermeiro em um serviço hospitalar de emergência. 2010. 136fls. Dissertação (Mestrado em Enfermagem) - Escola de Enfermagem, Universidade Federal do Rio Grande do Sul, Porto Alegre, 2010.

SOUZA, A. C. C.; MUNIZ FILHA, M. J. M.; SILVA, L. F.; MONTEIRO, A. R. M.; FIALHO, A. V. M. Formação do enfermeiro para o cuidado: reflexões da prática profissional. Revista Brasileira de Enfermagem, Brasília, v. 59, n. 6, p. 805-807, nov./dez. 2006.

SOUZA, M. T.; SILVA, M. D.; CARVAHO, R. de. Revisão integrativa: o que é e como fazer. Einstein, São Paulo, v. 8, n. 1, p. 102-106, jan./mar. 2010.

URSI, E. S. Prevenção de lesões de pele no perioperatório: revisão integrativa da literatura. 2005. 128 fls. Dissertação (Mestrado em Enfermagem). Universidade de São Paulo, Escola de Enfermagem de Ribeirão Preto, Ribeirão Preto. 2005.

VALENTIM, M. R. S.; SANTOS, M. L. S. C. Políticas de saúde em emergência e a enfermagem. Revista Enfermagem UERJ, Rio de Janeiro, v. 17, n. 2, p. 285-289, abr./jun. 2009. 